\title{
Artificial intelligence and radiology: Combating the COVID-19 conundrum
}

\section{Mayur Pankhania}

Sahyog Imaging Centre, Department of Radiodiagnosis, PDU Medical College and Government Hospital, Rajkot, Gujarat, India

Correspondence to: Mayur Pankhania, Sahyog Imaging Centre, Department of Radiodiagnosis, PDU Medical College and Government Hospital, Rajkot, Gujarat, India. E-mail: mayurpankhania@gmail.com

\begin{abstract}
The COVID-19 pandemic has necessitated rapid testing and diagnosis to manage its spread. While reverse transcriptase polymerase chain reaction (RT-PCR) is being used as the gold standard method to diagnose COVID-19, many scientists and doctors have pointed out some challenges related to the variability, accuracy, and affordability of this technique. At the same time, radiological methods, which were being used to diagnose COVID-19 in the early phase of the pandemic in China, were sidelined by many primarily due to their low specificity and the difficulty in conducting a differential diagnosis. However, the utility of radiological methods cannot be neglected. Indeed, over the past few months, healthcare consultants and radiologists in India have been using or advising the use of high-resolution computed tomography (HRCT) of the chest for early diagnosis and tracking of COVID-19, particularly in preoperative and asymptomatic patients. At the same time, scientists have been trying to improve upon the radiological method of COVID-19 diagnosis and monitoring by using artificial intelligence (Al)-based interpretation models. This review is an effort to compile and compare such efforts. To this end, the latest scientific literature on the use of radiology and Al-assisted radiology for the diagnosis and monitoring of COVID-19 has been reviewed and presented, highlighting the strengths and limitations of such techniques.
\end{abstract}

Key words: Artificial intelligence; COVID-19; coronavirus; HRCT; radiology

\section{Introduction}

The world has been grappling with a new viral disease-COVID-19. COVID-19, caused by severe acute respiratory syndrome coronavirus-2 (SARS-CoV-2), most commonly presents itself with fever, cough, and fatigue among other symptoms. ${ }^{[1]}$ As of June 17, 2020, there were around 8.1 million total cases of COVID-19 in the world, with 440,290 deaths. ${ }^{[2]}$

Real-time reverse transcriptase polymerase chain reaction (RT-PCR) for viral nucleic acid has been established as the gold standard diagnostic test for COVID-19

\begin{tabular}{|l|l|}
\hline \multicolumn{2}{|c|}{ Access this article online } \\
\hline Quick Response Code: & \\
\cline { 1 - 2 } & Website: \\
& www.ijri.org \\
\cline { 2 - 3 } & DOI: \\
&
\end{tabular}

although some scientists have raised concerns over this method. ${ }^{[3]}$ The sensitivity of the RT-PCR test has varied over time and among test centers. For instance, Fang et al. reported a sensitivity of $71 \%$ for the RT-PCR test, while the University of Washington Medicine in the US, found it to be $95 \%-97 \% .{ }^{[4,5]}$ Another study reported that the use of pharyngeal swab specimens for RT-PCR tests yields variable results, and this method alone should not be used to make decisions about known or suspected COVID-19 patients. ${ }^{[6]}$ Meanwhile, many countries are still struggling to conduct

This is an open access journal, and articles are distributed under the terms of the Creative Commons Attribution-NonCommercial-ShareAlike 4.0 License, which allows others to remix, tweak, and build upon the work non-commercially, as long as appropriate credit is given and the new creations are licensed under the identical terms.

For reprints contact: WKHLRPMedknow_reprints@wolterskluwer.com

Cite this article as: Pankhania M. Artificial intelligence and radiology: Combating the COVID-19 conundrum. Indian J Radiol Imaging 2021;31:S4-10.

Received: 18-Jul-2020

Accepted: 21-Sep-2020

Revised: 27-Aug-2020 Published: 23-Jan-2021 
sufficient RT-PCR tests because of the exorbitant cost of the reagents required..$^{[7-9]}$

In the initial stage of the pandemic, chest computed tomography (CT) was approved as a diagnostic method for COVID-19. ${ }^{[4]}$ Lack of a sufficient number of RT-PCR kits for diagnostic labs with an unprecedented increase in the number of patients necessitated the use of chest CT as a diagnostic approach, particularly in China when the spread of the disease was in its early stages. ${ }^{[4]}$ In fact, CT had proven to be a highly effective diagnostic approach at the time, with a sensitivity of $98 \%$, compared to $71 \%$ for RT-PCR. ${ }^{[5,10]}$ Another study that used chest CT recordings reported a sensitivity of $97 \%$, though the specificity and accuracy values were only $25 \%$ and $68 \%$, respectively. ${ }^{[11]}$ Over the past few months, several physicians in India have also been using or recommending high-resolution CT (HRCT) of the chest for early diagnosis and tracking of COVID-19, particularly in preoperative and asymptomatic patients. ${ }^{[12,13]}$

However, in March 2020, the American College of Radiology (ACR) recommended that radiological findings should not be used for preliminary diagnosis of COVID-19. [14] The reasons cited were as follows: (1) most of the studies describing the efficacy of radiological methods for COVID-19 diagnosis had come from China, thus restricting the data to one geographical location; (2) nonspecificity of radiological discoveries as clinically, the disease manifests in ways similar to some other respiratory diseases of viral origin; (3) As ACR's recommendations were made keeping in mind the state of affairs in the US, it was important to use more specific methods of diagnosis (RT-PCR) because of a large number of influenza cases in the country at that time of the year. ${ }^{[14-16]}$

Several studies have expanded COVID-19 radiology literature since the ACR's recommendations were published. Reports have not only improved in terms of geographical coverage but also the accuracy of interpretation. Moreover, artificial intelligence (AI) models have been created or adapted to aid radiologists in determining COVID-19 diagnosis and progression using radiological evidence. One study has even shown that the use of AI for interpreting radiological data improves the diagnosis of COVID-19 compared to when radiologists draw conclusions without an AI-based aid. ${ }^{[17]}$ The objective of this review is to summarize the latest literature that describes the use of AI-assisted radiology in the diagnosis and monitoring of COVID-19. The merits and demerits of these techniques have also been discussed.

\section{Methods}

We performed a systematic literature search, obtained the latest scientific literature on the use of radiology and AI-assisted technology for the diagnosis and monitoring of COVID-19, and presented the data extracted in this review. Articles available as preprints or published in online archives were not excluded from this review due to the contribution of such reports to the evolving impact of AI on radiology in the COVID-19 era.

\section{Radiological observations in COVID-19 patients}

Both HRCT scans and X-ray images of the chest have been used by radiologists to diagnose or monitor COVID-19. HRCT scans of the chest in COVID-19 patients are characterized by bilateral and peripheral ground-glass opacities (GGO), predominantly in the lower lobe ${ }^{[18-20]}$ [Figure 1]. Additionally, multiple plaque shadows, interlobular septal thickening and crazy pavingpatterns, lung consolidation, interstitial changes in the peripheral lung and the subpleural, and a reversed "halo" pattern are also found. ${ }^{[15,18,19,21-23]}$ These features distinguish COVID-19 from a healthy human or patients with pneumonia caused by other bacterial or viral infections. General features such as volume, shape, density of the lung lesions are also identified and compared for the identification of COVID-19. ${ }^{[2]}$ In chest radiographs, COVID-19 patients exhibit bilateral lower zone- and peripherally-predominant lung consolidation as well as hazy opacities. ${ }^{[15]}$

There are significant commonalities between the radiological features observed in COVID-19 and other forms of pneumonia, particularly those of viral origin. Ground-glass opacities, consolidation, and interlobular septal thickening are also observed in HRCT scans of the chest from patients with other types of viral pneumonia. ${ }^{[19]}$ Similarly, changes in the pulmonary interstitial space and alveolar wall edema are common characteristics in the chest radiographs of pneumonia caused by different viruses. ${ }^{[19]}$ However, some differences have still been observed. COVID-19 can be distinguished from H1N1 flu because of the small ground glass density shadow observed in chest CTs obtained even in the early stages of

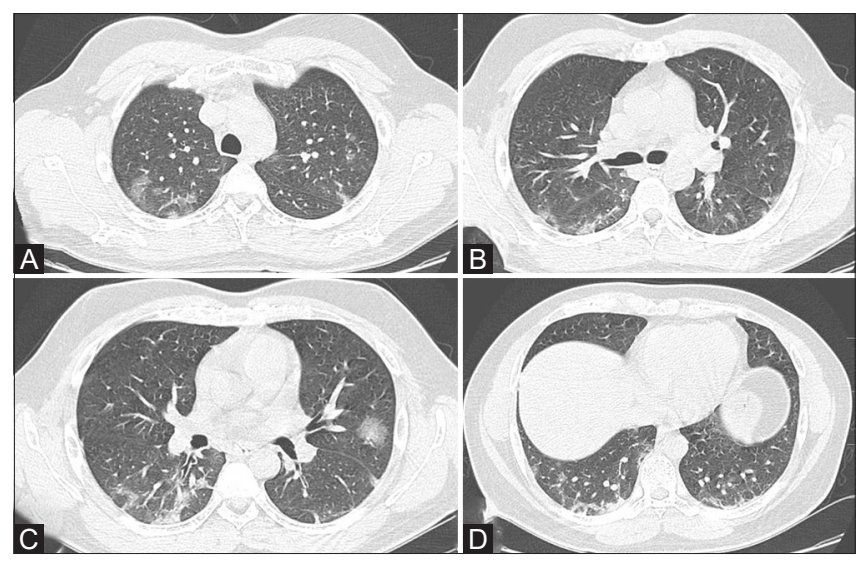

Figure 1 (A-D): (A-D) A 37-year-old male patient presented with low-grade fever and shortness of breath for 4 days. HRCT Chest shows patchy ground-glass opacities predominantly peripheral and basal in distribution. COVID-19 Infection 
COVID-19 pneumonia. ${ }^{[19,23]}$ Similarly, pneumonia caused by the human parainfluenza virus causes the appearance of peribronchial nodules, consolidation, and a centrally distributed lesion, while COVID-19 pneumonia shows a characteristic subpleural distribution. ${ }^{[19,22,23]}$ Moreover, crazy paving is not typically observed in other kinds of viral pneumonia.

The characteristic features are more likely to be observed in patients after the onset of clinical symptoms. Some features, such as ground-glass opacities, may be observed before the onset too. ${ }^{[15,25]}$ Thus, clinical symptoms and appearance of changes in radiological data are not always synchronized in COVID-19, which makes it challenging to use only radiological data to confirmedly diagnose COVID-19. ${ }^{[19]}$ HRCT chest helps in the identification and diagnosis of the cases with false negative pathological test results [Figure 2]. Radiologists not only help in triage of the patients; they also decide the severity of the disease by documenting Chest CT Severity Score and predict worsening and improvement in a follow-up patient. It is indeed a problem solver. ${ }^{[23]}$

Why do we need AI, especially for the COVID-19 diagnosis? A radiologist's experience is an important currency when it comes to making conclusive radiological observations. Some radiologists may have more experience (that is, they may have spent more time or handled more cases) diagnosing a given disease or abnormality with imaging, while others may not have had the opportunity to do so. Therefore, diagnoses based on radiological methods such as CT and X-ray can vary widely from one radiologist to another. The challenge is further increased when radiology is used to diagnose a disease like COVID-19 because many visual features of COVID-19 are similar to other lung illnesses as discussed earlier. Xu et al. reported that the sensitivity of COVID-19 diagnosis from CT scans by experienced radiologists ( $\geq 4$ years) in a Wuhan hospital ranged between $55 \%$ and $93 \%$. The specificity varied from $79 \%$ to $97 \% .{ }^{[21]}$ The relative novelty of our understanding



Figure 2 (A-I): (A-C) A 30-year-old male patient presented with low-grade fever and shortness of breath since a week with RT-PCR negative status showinga small patchy area of ground-glass opacity in left lower lobe. (D-I) Follow-up HRCT shows extensive ground-glass opacities in throughout both lungs, predominantly peripheral and basal in distribution. COVID-19 Infection 
of the lung radiological features associated with COVID-19 has led to such wide variations in their interpretation. Moreover, some of these visual features are not exclusive to COVID-19. ${ }^{[21]}$ As our knowledge about COVID-19 continues to evolve, AI has the power to help radiologists interpret and get acquainted with these differences between COVID-19 and other similar illnesses. Indeed, a study by Bai et al. has shown that radiologists assisted by AI achieved greater success in accurately diagnosing COVID-19 than when AI was not used. ${ }^{[17]}$ In this study, the accuracy increased from $85 \%$ to $90 \%$ with the aid of AI, while the sensitivity improved from $79 \%$ to $88 \%$ and the specificity changed from $88 \%$ to $91 \%$.

\section{Use of AI by radiologists during the COVID-19 crisis}

Several AI models have been developed and tested for their ability to identify COVID-19 amid chest radiographs and CT scans representing healthy population, COVID-19 patients, and patients suffering from other kinds of pneumonia. The pertinent features of some of these AI models have been summarized in Table 1.

In one study, Li et al. have created a deep learning model called COVID-19 Detection Neural Network (COVNet). ${ }^{[26]}$ The extensive study included 4,536 3D chest CT exams (from 3,322 patients), of which $30 \%$ were conducted for COVID-19, 40\% for community-acquired pneumonia, and the remaining $30 \%$ for nonpneumonia patients. The COVID-19 cases included in this study were all confirmed positive by the RT-PCR method. The program developed in the study successfully extracts $2 \mathrm{D}$ local and 3D global features from the CT radiographs. The method has an overall sensitivity of $90 \%$ and specificity of $96 \%$ for detecting COVID-19.

Gozes et al. also used CT recordings to identify COVID-19 cases with the help of AI. ${ }^{[27]}$ They employed a ResNet50 model for binary classification of COVID-19 and normal CT scans. However, their training data set also came from a Chinese cohort, with 829 recordings from COVID-19 patients and 1,036 healthy recordings. While the COVID-19 data used to test the program came from the Chinese patients only, the study used non-COVID-19 data from patients in China and the US separately to arrive at different accuracies and specificities. The authors of this study altered the threshold, or the fraction of CT slices that were detected positive for COVID-19, to determine the maximum sensitivity and specificity that the program could achieve. The program registered a sensitivity of $98.2 \%$, with a corresponding specificity of $92.2 \%$ for the all-Chinese cohort when a threshold of $1.1 \%$ was used. With a threshold of $1.9 \%$, the sensitivity decreased to $96.4 \%$ while the specificity of detection increased to $98 \%$. When non-COVID-19 data from US patients were included, a sensitivity values of $98.2 \%$ (threshold $=1 \%$, specificity $=91.8 \%$ ) and $94.6 \%$ (threshold $=4.3 \%$, specificity $=98 \%$ ) were achieved.

In a similar study, Bai et al.used Efficient Net architecture to train their classification model so that COVID-19 and non-COVID-19 CT images could be separated. ${ }^{[17]} \mathrm{Xu}$ et al. also used chest $\mathrm{CT}$ scans and classified them into healthy or one of three types of pneumonia (COVID-19, bacterial, and viral) using a 14-layered 3D Densenet convolutional neural network (CNN). ${ }^{[21]}$

Chest radiographs have also been used to develop AI models that can diagnose COVID-19. Ucar et al. used the Bayesian optimization-based Squeeze Net model to analyze chest radiographs to distinguish among healthy, COVID-19, and other pneumonia patients. ${ }^{[28]}$ This method allowed for the augmentation of small-sized imaging datasets before training and testing. All available images were divided into three classes - training, validation, and

Table 1: Al models developed for identification of COVID-19 through radiological data

\begin{tabular}{|c|c|c|c|c|}
\hline Type of model & Type of data & Level of classification (data sets included) & $\begin{array}{l}\text { Mean accuracy of } \\
\text { COVID-19 identification }\end{array}$ & Reference \\
\hline $\begin{array}{l}\text { Convolutional Neural Network or CNN } \\
\text { (ResNet50) (COVNet) }\end{array}$ & CT Scans & $\begin{array}{l}\text { Ternary (COVID-19, community-acquired } \\
\text { pneumonia, nopneumonia) }\end{array}$ & - & Li et al. ${ }^{[26]}$ \\
\hline Deep Neural Network (SqueezeNet) & Chest Radiographs & Ternary (COVID-19, other pneumonia, healthy) & $98.3 \%$ & Ucar et al. ${ }^{[28]}$ \\
\hline CNN & Chest Radiographs & Ternary (COVID-19, other pneumonia, healthy) & $95.7 \%$ & Al-Asfoor et al. ${ }^{[29]}$ \\
\hline CNN based on 3D-DenseNet & CT Scans & $\begin{array}{l}\text { Quaternary (COVID-19, viral pneumonia, } \\
\text { bacterial pneumonia, healthy) }\end{array}$ & $72 \%-97 \%$ & Xu et al..$^{[21]}$ \\
\hline $\begin{array}{l}\text { CNN (Inception-V3) + Deep neural } \\
\text { network }\end{array}$ & Chest Radiographs & $\begin{array}{l}3 \text { comparisons made: binary (COVID-19 and } \\
\text { other pneumonia); ternary (COVID-19, other } \\
\text { pneumonia and healthy); quaternary (COVID-19, } \\
\text { bacterial pneumonia, viral pneumonia, healthy) }\end{array}$ & $\begin{array}{c}100 \% \text { (for binary), } 85 \% \\
\text { (for ternary) and } 76 \% \text { (for } \\
\text { quaternary) }\end{array}$ & Tsiknakis et al. ${ }^{[31]}$ \\
\hline CNN (ResNet50) & Chest Radiographs & Binary (COVID-19 and healthy) & $98 \%$ & Narin et al. ${ }^{[30]}$ \\
\hline CNN (Inception-V3) & Chest Radiographs & Binary (COVID-19 and healthy) & $97 \%$ & Narin et al..$^{[30]}$ \\
\hline CNN (Inception-ResNetV2) & Chest Radiographs & Binary (COVID-19 and healthy) & $87 \%$ & Narin et al. ${ }^{[30]}$ \\
\hline CNN (ResNet50) & CT Scans & Binary (COVID-19 and non-COVID-19) & $98.2 \%$ & Gozes et al. ${ }^{[27]}$ \\
\hline Deep Neural Network (EfficientNet B4) & CT Scans & Binary (COVID-19 and non-COVID-19) & $96 \%$ & Bai et al. ${ }^{[17]}$ \\
\hline
\end{tabular}


testing. The validation step helped update the Bayesian optimization process to improve the model and obtain the best possible network model for the testing dataset. In another study, Al-Asfoor et al.used 1,585 normal, 123 COVID-19, and 4275 pneumonia chest radiographs to train and test their 22-layered convolutional neural network (CNN) model. ${ }^{[29]}$

Narin et al. used 50 chest radiographs from COVID-19 patients and 50 normal images and analyzed them using the ResNet50, Inception-V3, and Inception-ResNetV2 models. ${ }^{[30]}$ The ResNet50 model had the highest accuracy (98\%) while the Inception-ResNetV2 model had the lowest (87\%). Meanwhile, the Inception-V3 model had an accuracy of $97 \%$. In comparison, another Inception-V3 model developed by Tsiknakis et al.was shown to perform better with a greater number of images used for transfer learning. The AI program developed by Tsiknakis et al. ${ }^{[3]}$ also interprets chest radiographs and determines if they are representative of COVID-19 or other forms of pneumonia. Their study collated 137 multinational COVID-19 chest radiographs (from Italy, Mexico, Argentina, India, and others) to develop the proposed AI model. The other groups had 150 randomly chosen images each. The study used the Inception-V3 model for binary, ternary as well as quaternary analyses, with an accuracy of $100 \%$ for the binary comparison.

The studies described here show that it is indeed possible to tune the parameters of AI models to improve the accuracy, sensitivity, and specificity of diagnosing COVID-19 through radiological data.

\section{Interpretability of AI-based programs}

A pervasive challenge to using $\mathrm{AI}$ in radiology is the interpretability of the results and interpretations provided by AI-based programs. Interpretability is the human understanding of the relationship between the extraction of radiological/visual features by the AI program and the subsequent predictions it makes. ${ }^{[32]}$ If the end-users are unable to understand how a deep learning AI algorithm draws medical conclusions from radiological images, their trust in such programs would be deeply affected. Some recent studies on the use of $\mathrm{AI}$ in the interpretation of radiological data from COVID-19 patients have also attempted to improve the interpretability of their method so that the end-users can have a greater trust in the derived conclusions. For example, Tsiknakis et al. incorporated attention maps as an interpretability measure for each prediction made by their program.

\section{The scenario in India}

In India, the importance of AI in detecting COVID-19 from chest radiographs images has already led to the development of new solutions. Companies providing AI technology for radiology departments have already launched solutions for diagnosing and managing COVID-19. has offered an
AI-based solution called qXR, which helps monitor the daily progression of COVID-19 in patients. ${ }^{[33]}$ This solution measures the percentage of COVID-19-infectedlung volume through chest radiographs. The AI program identifies COVID-19 features such as ground-glass infiltrates and lung consolidation. It further categorizes the patients as low-, medium-, or high-risk based on these data. This solution is already in use in many hospitals around the world, including India. Another company, the UK-based Behold.ai, has launched its "instant triage" solution that also uses AI to interpret chest radiographs. This solution has now been implemented by some hospitals in India. ${ }^{[34]}$ Both technologies enable the identification of COVID-19 cases in under a minute.

\section{Limitations and strengths}

In a "Machine Intelligence in Healthcare" workshop co-hosted by the National Institutes of Health (NIH) National Centre for Advancing Translational Sciences (NCATS) along with several other agencies in July 2019, experts agreed that AI is transforming the landscape of biomedical research and medical diagnostics. ${ }^{[3]} \mathrm{AI}$-based solutions help both physicians and patients to make better healthcare decisions, thereby improving the efficacy of the healthcare system. However, in the context of COVID-19 diagnosis and monitoring, the limitations and strengths of AI-based radiological solutions are still being explored and discovered.

Most studies using AI to analyze and interpret radiological images for the diagnosis of COVID-19 are limited by the geographical source of patient data. Variations have been noted when CT scans from a single region or country are used against the pooling of data from multiple locations. For example, Xu et al. developed a model which had a sensitivity of $97.5 \%$ when used against CT data obtained from Tongji Hospital in Wuhan, but only $72 \%$ for cases from Wuhan Union Hospital. ${ }^{[21]}$ Thus, there is a lack of consistency in performance for some of the AI-based models developed to aid COVID-19 diagnosis.

Additionally, a highly sensitive and specific AI-based model for diagnosing COVID-19 requires training on a large amount of well-annotated radiological data. The studies conducted so far have shown large variability in this area, with the number of images used for training ranging from around 150 to more than 4,000 . Future studies must increase the size of the data sets used to train the AI programs for COVID-19 diagnosis. Given that COVID-19 is a newly identified disease for which research, data collection, and annotation is still underway, developing appropriately trained ML algorithms may take some time. Xu et al. proposed a platform where decentralized radiological data from CT scans all over the world could be used freely by AI scientists and radiologists to devise an accurate model for COVID-19 diagnosis. The platform, called the Unified CT-COVID AI Diagnostic Initiative (UCADI), 
would help collate a large amount of imaging data for training any new AI-based programs. ${ }^{[21]}$

Given its limitations, some radiologists are not in agreement with the idea that AI could replace human interpretation. ${ }^{[36]}$ Radiologists and other doctors in many parts of the world have strongly voiced that radiological methods, even with the use of artificial intelligence, cannot replace laboratory tests such as RT-PCR for the diagnosis of COVID-19. ${ }^{[14,37]}$ However, doctors have recommended the use of CT scans for monitoring disease progression and remission in noncritical but confirmed cases of COVID-19. ${ }^{[19]}$ Further, in the cases where molecular diagnostic tests are inconsistent with symptomatic observations, or when there are complications or critical illnesses, radiological approaches may be used as a secondary or alternate approach for diagnosis..$^{[18,38,39]}$ Additionally, some doctors have recommended the use of radiological methods as an assisting diagnostic approach as well as a follow-up strategy in pediatric patients too. ${ }^{[40]}$

Even when chest radiological approaches are not used for diagnosis of COVID-19, they have an important role to play in understanding the pathological changes in the lungs of COVID-19 patients. ${ }^{[18]}$ Quantifying the fraction of the lung parenchyma that is affected by COVID-19-like pathological changes can be useful in monitoring the progression of the disease ${ }^{[36]}$ Moreover, the use of AI in radiology enables a quantitative scoring through programs that can be easily revised and updated as the disease and our knowledge of it evolves over time. ${ }^{[18,27]}$

The strength of obtaining and interpreting radiological data for COVID-19 is that it can also be used to predict mortality. In one study, Yuan et al. used a 3-point scale to score CT data, where a score of 1 corresponded to normal attenuation, 2 to ground-glass attenuation, and 3 to consolidation. A 4-point scale was additionally used to score the extent of abnormalities in the lung parenchyma. Using a combination of these scores, they determined a net score to predict COVID-19 mortality with a sensitivity of $85.6 \%$ and a specificity of $84.5 \% .{ }^{[4]}$ While this study did not use AI, future studies that employ AI for calculating mortality scores could help doctors decide the right course of treatment for different COVID-19 patients.

Overall, despite their limitations, AI-based radiological methods of COVID-19 diagnosis offer many advantages: (1) reduced processing and diagnosis time compared to both the RT-PCR method as well as the human interpretation of radiological data, (2) alternative diagnostic method in the absence of resources to conduct RT-PCR tests for all suspected COVID-19 cases, (3) monitoring the progression of the disease, and (4) monitoring the effectiveness of therapeutic approaches.

\section{Conclusion}

While real-time RT-PCR for viral nucleic acid has been established as the gold standard diagnostic test for COVID-19; however, HRCT Chest is valuable for the early diagnosis of COVID-19, particularly for those patients with a negative RT-PCR.HRCT of the chest is a fast replacing pathological test for the early diagnosis of COVID-19 and proving to be a game-changer in populous countries such as India where the availability of RT-PCR tests is low, and the time taken for diagnosis is higher. However, AI-based models for radiology-based COVID-19 diagnosis still need to be improved with larger, well-annotated training datasets. This improvement will happen as we keep learning more about this new disease and its radiological signatures.In the meantime, AI-based models can become usefultools to monitor COVID-19 progression through follow-up imaging. With the appropriate use of AI, radiological methods hold the potential screen toolsto help a large number of people efficiently and quickly, enable the monitoring of COVID-19 progression, and help in the preliminary diagnosis of noncritical suspected patients. Even though AI cannot replace the human radiologist, it could become a trusted aid to help us defeat the COVID-19 conundrum swiftly and completely.

Financial support and sponsorship

Nil.

Conflicts of interest

There are no conflicts of interest.

\section{References}

1. Huang C, Wang Y, Li X, Ren L, Zhao J, Hu Y, et al. Clinical features of patients infected with 2019 novel coronavirus in Wuhan, China. Lancet 2020;395:497-506.

2. WHO. Coronavirus disease (COVID-19) - Situation report 149: World Health Organization; 2020. Available from: https://www.who.int/docs/default-source/ coronaviruse/situation-reports/20200617-covid-19-sitrep-149. pdf?sfvrsn=3b3137b0_8. [Updated Jun 17; Cited on 2020 Jun 18].

3. Dramé M, Teguo MT, Proye E, Hequet F, Hentzien M, Kanagaratnam L, et al. Should RT-PCR be considered a gold standard in the diagnosis of Covid-19? J Med Virol 2020.doi: 10.1002/jmv.26228.

4. Mossa-Basha M, Meltzer CC, Kim DC, Tuite MJ, Kolli KP, Tan BS. Radiology department preparedness for COVID-19: Radiology scientific expert review panel. Radiology 2020;296: E106-12.

5. Fang Y, Zhang H, Xie J, Lin M, Ying L, Pang P, et al. Sensitivity of chest CT for COVID-19: Comparison to RT-PCR. Radiology 2020;296:E115-7.

6. Li Y, Yao L, Li J, Chen L, Song Y, Cai Z, et al. Stability issues of RT-PCR testing of SARS-CoV-2 for hospitalized patients clinically diagnosed with COVID-19. J Med Virol2020;92:903-8.

7. Maxmen A. How poorer countries are scrambling to prevent a coronavirus disaster. Nature 2020;580:173-74.

8. Ramdas K, Darzi A, Jain S. 'Test, re-test, re-test': Using inaccurate tests to greatly increase the accuracy of COVID-19 testing. Nat Med 2020;26:810-11. 
9. Wild S. African countries scramble to ramp up testing for COVID-19. Scientific American; 2020. Available from: https://www. scientificamerican.com/article/african-countries-scramble-to-rampup-testing-for-covid-19/.

10. Xue H, Jin Z. The appropriate position of radiology in COVID-19 diagnosis and treatment-current status and opinion from China. Chin J AcadRadiol 2020. doi: 10.1007/s42058-020-00030-6.

11. Ai T, Yang Z, Hou H, Zhan C, Chen C, Lv W, et al. Correlation of chest CT and RT-PCR testing for Coronavirus Disease 2019 (COVID-19) in China: A report of 1014 cases. Radiology 2020;296:E32-40. doi: 10.1148/radiol.2020200642.

12. Shastri P. Gujarat doctors use CT scan as Covid test, 73 turn out to be positive. Times of India 2020.

13. Kohli A. Can imaging impact the coronavirus pandemic? Indian J Radiol Imaging 2020;30:1-3.

14. ACR recommendations for the use of chest radiography and computed tomography (CT) for suspected COVID-19 infection [Internet]. American College of Radiology; 2020; March 22. Available from: https://www.acr.org/ Advocacy-and-Economics/ACR-Position-Statements/Recomm endations-for-Chest-Radiography-and-CT-for-Suspected-CO VID19-Infection.

15. Czawlytko C, Hossain R, White CS. COVID-19 diagnostic imaging recommendations. Appl Radiol 2020;49:10-5.

16. Kooraki S, Hosseiny M, Myers L, Gholamrezanezhad A. Coronavirus (COVID-19) outbreak: What the department of radiology should know. J Am Coll Radiol2020;17:447-51.

17. Bai HX, Wang R, Xiong Z, Hsieh B, Chang K, Halsey $\mathrm{K}$, et al. Artificial intelligence augmentation of radiologist performance in distinguishing COVID-19 from pneumonia of other etiology on chest CT. Radiology 2020;296(3):E156-65.

18. Kundu S, Elhalawani H, Gichoya JW, Kahn Jr CE. How might AI and chest imaging help unravel COVID-19's mysteries? RadiolArtifIntell 2020. doi: 10.1148/ryai.2020200053.

19. Chen H, Ai L, Lu H, Li H. Clinical and imaging features of COVID-19. Radiol Infect Dis 2020;7:43-50.

20. Hani C, Trieu NH, Saab I, Dangeard S, Bennani S, Chassagnon G, et al. COVID-19 pneumonia: A review of typical CT findings and differential diagnosis. Diagn Interv Imaging 2020;101:263-8.

21. Xu Y, Ma L, Yang F, Chen Y, Ma K, Yang J, et al. A collaborative online AI engine for CT-based COVID-19 diagnosis. medRxiv 2020. doi:https://doi.org/10.1101/2020.05.10.20096073.

22. Carotti M, Salaffi F, Sarzi-Puttini P, Agostini A, Borgheresi A, Minorati $\mathrm{D}$, et al. Chest $\mathrm{CT}$ features of coronavirus disease 2019 (COVID-19) pneumonia: Key points for radiologists. Radiol Med 2020;125:636-646.

23. Chua F, Armstrong-James D, Desai SR, Barnett J, Kouranos V, Kon OM, et al. The role of CT in case ascertainment and management of COVID-19 pneumonia in the UK: Insights from high-incidence regions. Lancet Respir Med 2020;8:438-40.

24. Tripathi SC, Deshmukh V, Patil A, Tripathy JP. COVID-19 diagnostic multiplicity and its role in community surveillance and contol.Infez Med 2020;28(suppl 1):18-28.

25. Wang Y, Dong C, Hu Y, Li C, Ren Q, Zhang X, et al. Temporal changes of CT findings in 90 patients with COVID-19 pneumonia: A longitudinal study. Radiology 2020;296:E55-64.

26. Li L, Qin L, Xu Z, Yin Y, Wang X, Kong B, et al. Artificial intelligence distinguishes COVID-19 from community acquired pneumonia on chest CT. Radiology 2020.doi: 10.1148/radiol.2020200905.
27. Gozes O, Frid-Adar M, Greenspan H, Browning PD, Zhang $\mathrm{H}$ Ji W, et al. Rapid AI development cycle for the coronavirus (COVID-19) pandemic: Initial results for automated detection \& patient monitoring using deep learning CT image analysis. arXiv preprint arXiv:200305037 2020

28. Ucar F, Korkmaz D. COVIDiagnosis-Net: Deep Bayes-SqueezeNet based diagnostic of the coronavirus disease 2019 (COVID-19) from X-Ray Images. Med Hypotheses 2020;140:1-12. doi: 10.1016/j. mehy.2020.109761.

29. Al-Asfoor M, Abed MH. Deep learning approach for COVID-19 diagnosis using X-ray images. Research Square Preprints 2020. doi: 10.21203/rs.3.rs-31278/v1

30. Narin A, Kaya C, Pamuk Z. Automatic detection of coronavirus disease (covid-19) using $x$-ray images and deep convolutional neural networks. arXiv preprint arXiv: 200310849, 2020.

31. Tsiknakis N, Trivizakis E, Vassalou EE, Papadakis GZ, Spandidos DA, Tsatsakis A, et al. Interpretable artificial intelligence framework for COVID-19 screening on chest X-rays. Exp Ther Med 2020;20(2):727-35.

32. Reyes M, Meier R, Pereira S, Silva CA, Dahlweid F-M, Tengg-Kobligk HV, et al. On the interpretability of artificial intelligence in radiology: Challenges and opportunities. RadiolArtifIntell 2020;2:e190043.

33. Bora G. Qure.ai can detect Covid-19 lung infections in less than a minute, help triage patients. Economic Times. 2020, April 30. Available from: https://economictimes.indiatimes.com/small-biz/ startups/newsbuzz/qure-ai-can-detect-covid-19-lung-infections-i n-less-than-a-minute-help-triage-patients/articleshow/75464679. cms.

34. Behold.ai. Behold.ai partners with Apollo Hospitals Group, India for rapid COVID-19 diagnosis using AI-based chest X-ray technology London, UK2020 [Updated on May 18]. Available from: https://behold.ai/behold-ai-partners-with-apollo-hospitalsgroup-india-for-rapid-covid-19-diagnosis-using-ai-based-chest -x-ray-technology/.

35. Cutillo CM, Sharma KR, Foschini L, Kundu S, Mackintosh M, Mandl KD. Machine intelligence in healthcare-perspectives on trustworthiness, explainability, usability, and transparency. NPJ Digit Med 2020;3:1-5.

36. Laghi A. Cautions about radiologic diagnosis of COVID-19 infection driven by artificial intelligence. Lancet Digit Health 2020;2:e225

37. Neri E, Miele V, Coppola F, Grassi R. Use of CT and artificial intelligence in suspected or COVID-19 positive patients: Statement of the Italian Society of Medical and Interventional Radiology. Med Radiol2020;125:505-8.

38. Rubin GD, Ryerson CJ, Haramati LB, Sverzellati N, Kanne JP, Raoof $\mathrm{S}$, et al. The role of chest imaging in patient management during the COVID-19 pandemic: A multinational consensus statement from the Fleischner Society. Chest 2020;158:106-16.

39. Rodrigues J, Hare S, Edey A, Devaraj A, Jacob J, Johnstone A, et al. An update on COVID-19 for the radiologist-A British society of thoracic imaging statement. Clin Radiol 2020;75:323-5.

40. Mathew RP, Jose M, Toms A. The role of non-contrast chest CT in suspected or confirmed coronavirus disease 2019 (COVID-19) pediatric patients. Indian J Pediatr 2020.doi: 10.1007/ s12098-020-03338-4.

41. Yuan M, Yin W, Tao Z, Tan W, Hu Y. Association of radiologic findings with mortality of patients infected with 2019 novel coronavirus in Wuhan, China. PLoS One 2020;15:e0230548. 